\title{
Diffuse Parenchymal Lung Diseases: 3D Automated Detection in MDCT
}

\author{
Catalin Fetita $^{1}$, Kuang-Che Chang-Chien ${ }^{1,4}$, Pierre-Yves Brillet ${ }^{1,2}$, \\ Françoise Prêteux ${ }^{1}$, and Philippe Grenier ${ }^{3}$ \\ ${ }^{1}$ Dept. ARTEMIS, INT, Groupe des Ecoles des Télécommunications, Evry, France \\ 2 AP-HP, Avicenne Hospital, Bobigny, France \\ ${ }^{3}$ Université Paris 6 and AP-HP, Pitié-Salpêtrière Hospital, Paris, France \\ ${ }^{4}$ National Chung Cheng University, Chia-yi, Taiwan, R.O.C
}

\begin{abstract}
Characterization and quantification of diffuse parenchymal lung disease (DPLD) severity using MDCT, mainly in interstitial lung diseases and emphysema, is an important issue in clinical research for the evaluation of new therapies. This paper develops a 3D automated approach for detection and diagnosis of DPLDs (emphysema, fibrosis, honeycombing, ground glass). The proposed methodology combines multiresolution image decomposition based on 3D morphological filtering, and graph-based classification for a full characterization of the parenchymal tissue. The very promising results obtained on a small patient database are good premises for a near implementation and validation of the proposed approach in clinical routine.
\end{abstract}

\section{Introduction}

Diffuse parenchymal lung diseases (DPLDs) include chronic obstructive lung disease which is defined by lung destruction (emphysema), and idiopathic interstitial pneumonias which are characterized by lung infiltrates (ground glass) and fibrosis.

High-resolution computed tomography (HRCT) with 1-mm-thick sections obtained at $10-\mathrm{mm}$ intervals has been widely accepted as the imaging reference standard for assessing DPLDs. Based on HRCT images, the diagnosis is done by analysing the different patterns of lung texture, and the severity of the disease can be evaluated by quotation of the extent of lesions. Nowadays, multidetector row CT (MDCT) generates isotropic volumetric high-resolution data and allows contiguous visualization of the lung parenchyma. MDCT tends to replace HRCT examinations since it allows creating three-dimensional reformatted images of excellent quality and significance.

Unfortunately, pathology features on the MDCT images sometimes would be subtle, especially in the early stage, and cause inter-observer variability even among the experienced radiologists. Hence, computer-aided diagnosis (CAD) is required for objective quantitative assessment of alterations in the lung [1. Several studies in the medical and technical literature have addressed the classification problem of the lung parenchyma. Without claiming an exhaustive analysis, these studies can be roughly divided into two categories, density-based and

N. Ayache, S. Ourselin, A. Maeder (Eds.): MICCAI 2007, Part I, LNCS 4791, pp. 825 833 2007.

(C) Springer-Verlag Berlin Heidelberg 2007 
texture-based. The principle of the density-based analysis is to investigate the relationship between a defined threshold value and the ratio of the lung tissue area/volume below this threshold [2. However, these methods cannot discriminate between several pathologies of the lung parenchyma occurring at the same time. In order to solve this problem, Blechschmidt et al. [3] defined an additional Bulae index which combines density measures with some texture-based approaches to quantify emphysema with concomitant fibrosis. The texture-based methods compute several measures in order to describe the CT attenuation characteristics. Then, a classifier (Bayesian, support vector machines, ...), performs an automated discrimination of the experimented samples. Malone et al. [4 proposed a texture-based approach to evaluate the pulmonary parenchyma from CT images. They split each image into grids with three block sizes, 4, 8, and 16 pixels, and computed 18 textural features for each block. Xu et al. [5] extended a run-length encoding method to three-dimensional (3D) data for volumetric texture analysis. Chabat et al. [6] adopted 13 features for extracting texture information from CT images. These features can be divided into three categories: $\mathrm{n}^{\text {th }}$ order statistical moments, spatial dependence of gray-scale distribution, and acquisition-length parameters. Recently, Xu et al. [78] used 3D texture features to enhance a previous two-dimensional (2D) classification system, the adaptive multiple feature method. The new system would be able to consider co-existing pathologies and to provide a simultaneous classification for differentiating between emphysema-like tissues found in normal smokers versus non-smokers.

In this paper we develop an original, fully-3D approach for automated detection of DPLDs in MDCT, based on the analysis of low-density patterns of the lung parenchyma. The proposed approach performs a multiresolution image data decomposition according to a specific morphological filtering. The result of the decomposition is synthesized into a hierarchic tree graph which nodes are analyzed in terms of textural and spatial relationship. Such analysis provides a classification of the decomposition patterns in normal and DPLD types.

The paper is organized as follows. Section 2 presents the image multiresolution decomposition scheme, and recalls the 3D morphological filter there considered. Section 3 describes and illustrates the generation of the descriptive tree graph and the performed classification analysis. The results are presented and discussed in Section 4 .

\section{Multiresolution Decomposition of Image Lung Parenchyma}

Lung parenchyma tissue can be roughly described as a collection of vascularized structures of tree-like topology and of various calibers decreasing with the subdivision order and crossing each other, namely the arterial, venous and tracheobronchial trees. From an image analysis point of view, such a multiple crossing, coupled with the influence of the MDCT acquisition protocol, results in a complex parenchymal texture which can be described and characterized by the distribution and the size of the low-density patterns delimited by the vascularized structures. 
Within normal tissue, the low-density patterns have a small size and are uniformly distributed (Fig. 1(a) . Such patterns change in the case of DPLDs due to tissue damages. Emphysema is characterized by larger, round-shape patterns of nearlyconstant low gray value, surounded by normal tissue (Fig. 1(b) . The appearance of emphysema/fibrosis patterns is similar, but their border is of a higher density than normal tissue. Honeycombing is characterized by agglomerations of several low-density patterns of similar size and dense thin or thick borders (Fig. 1(c)) Finally, ground glass tissue "infiltrates" the normal zones with medium-high opacities (Fig. $1(\mathrm{~d})$.

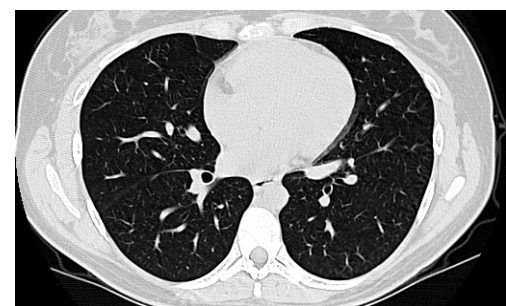

(a) Normal tissue.

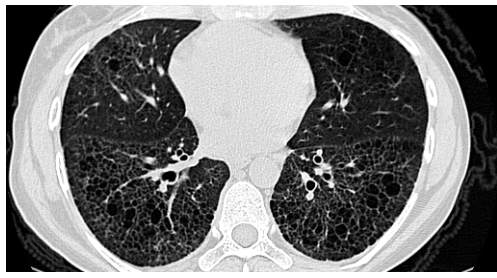

(c) Fibrosis/honeycombing.

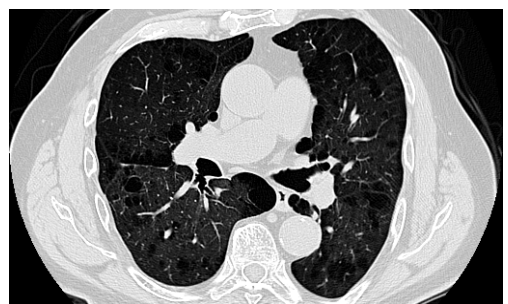

(b) Emphysema.

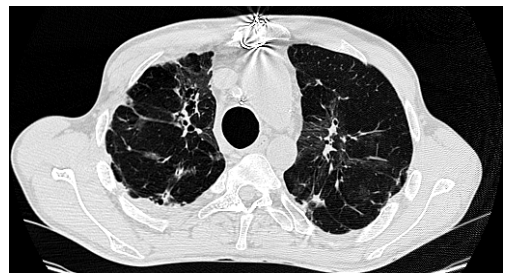

(d) Ground glass and emphysema.

Fig. 1. Some examples of MDCT axial images showing the patterns specific of different lung diseases

The idea exploited in this paper is the possibility to discriminate between normal and pathologic lung tissues by analyzing the size and distribution of, and the relationship between the low-density patterns. A one-dimensionnal schematic representation of the lung tissue, according to the previous remarks, is given in Fig. 2. Such "relief" representation will be exploited in the following in order to illustrate the principle of the developed approach.

In order to detect and analyze the different low-density patterns of the lung texture (local valleys on the lung "relief", Fig. 2) we have developed an image multiresolution decomposition scheme based on a 3D morphological filter, the sup-constrained connection cost introduced in [9]. Such a filter, denoted by $\mathcal{R C}_{f}^{n}$, will affect a function "relief" $f: \Re^{n} \rightarrow \Re$ by "filling in", at constant level, all local "basins" of $f$, of spatial extent smaller than $n$ and disconnected from larger "basins". Note that the considered filter do not modify the "basins" shape. They can be at most flooded if enclosed in larger "basins" of higher "walls". The 
increasing property of $\mathcal{R C}_{f}^{n}$ allows to apply such filter recursively, with increasing size, in order to progressively select all "basins" of a $f$ "relief".

The multiresolution decomposition scheme, illustrated in Fig. 3 for 4 levels of decomposition, exploits the $\mathcal{R C}_{f}^{n}$ properties and builds-up a hierarchic relief of the lung parenchyma, $g: \Re^{3} \rightarrow \Re$, as follows.

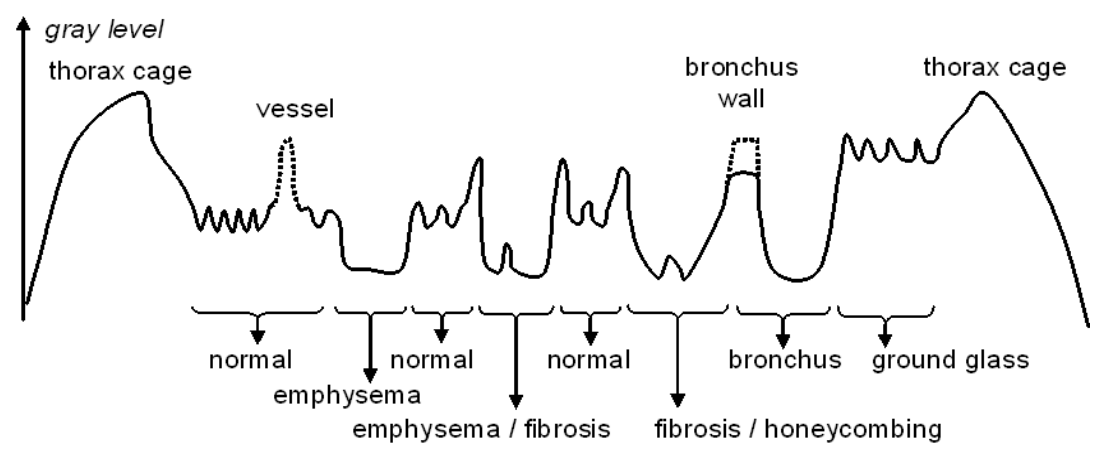

Fig. 2. 1-D schematic representation of normal and pathologic lung tissues. The dashed line symbolizes that a connectivity is possible at that level between the left and the right sides.

If $L$ denotes the maximum image value of the 3D thorax CT data, $n_{i}$ the filter size at level $i, n_{j}>n_{i} \forall j>i$, and $N_{\max }$ the maximum filter size, the decomposition procedure is given by:

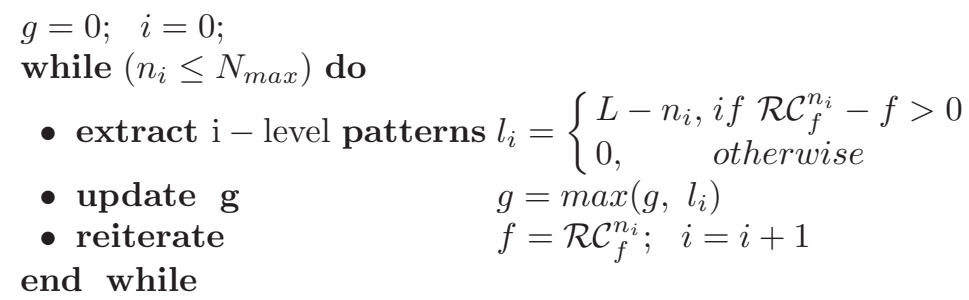

Finally, the eventual ground glass zones, which cannot be selected with the above procedure, are included as the lowest level of $g$. Their segmentation is based on a gray-scale reconstruction [10] of $\mathcal{R C}_{f}^{N_{\max }}$ with respect to its largest plateaux and suppression of the high-density thorax cage (Fig. 3).

Fig. 4 shows the corresponding axial cross-section images of the 3D hierarchic decomposition obtained with 10 resolution levels for the patients in Fig. 1 (histogram-equalized). Note that the descriptive information is free of highdensity tissues (vessels, thorax cage) and concerns only the lung parenchyma and the airway structure. A level of such decomposition informs on the size of the lung patterns at this level and also on a possible pathologic status (isolated larger patterns could point to disorders like emphysema/fibrosis/honeycombing). Conversely, patterns extracted at a decomposition level are independent of the 
native data values (for example, in Fig. 3, the 0-level patterns come from both normal and pathologic tissues).

By combining the information of the lung image decomposition (patterns size, $3 \mathrm{D}$ spatial and inter-level relationships) and the corresponding native image density values, a classification of the lung texture can be more confidently achieved. For an effective analysis, all this information is gathered in a 3D hierarchic descriptive graph built-up from the multi-level decomposition and presented in the following section.

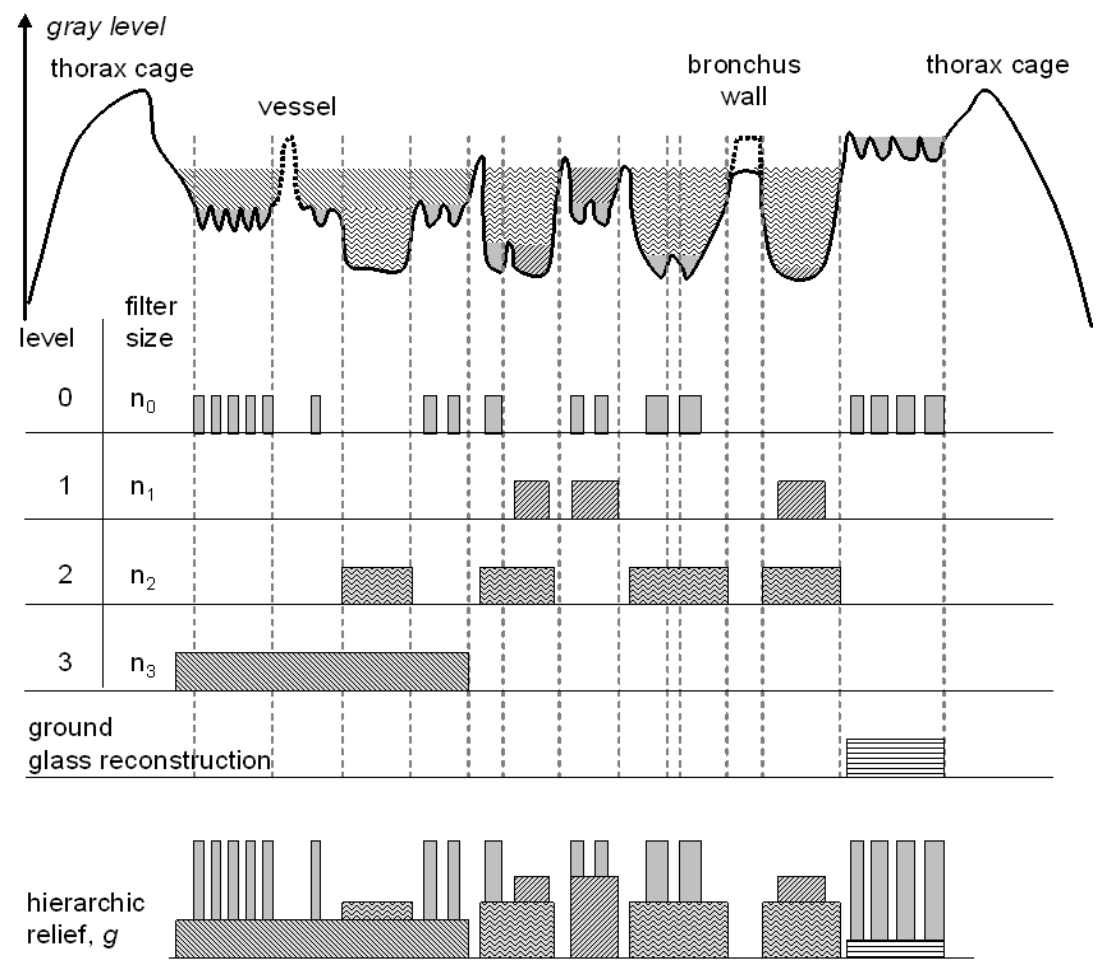

Fig. 3. Multiresolution decomposition of the schematic lung relief of Fig. 2

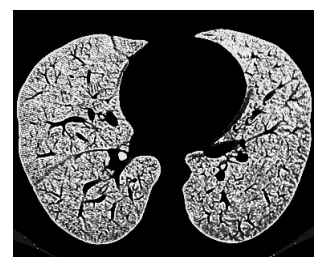

(a)

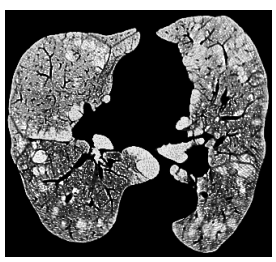

(b)

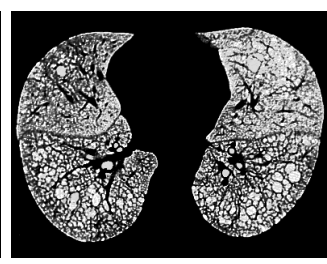

(c)

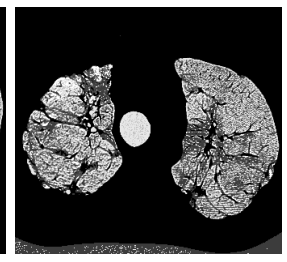

(d)

Fig. 4. Example of multiresolution hierarchic decomposition of data from Fig. 1 


\section{Hierarchic Classification of the Lung Tissue}

The objective is to obtain a compact, multi-valued description of the lung parenchyma based on the previous image decomposition and original data information. We shall show in the following that an information node can be associated with each pattern extracted at each resolution level of the decomposition and that a connectivity relationship can be established between such nodes, providing a descriptive tree graph.

Starting from the hierarchic relief $g$ (Fig. 3), the patterns of a level $i$ can be reconstructed by means of morphological operations, as follows. Using the notations of eq. 1

$i=0$;

while $\left(n_{i} \leq N_{\max }+1\right)$ do

- extract pattern subset of level i $\quad l_{i}^{\prime}=T_{L-n_{i}}^{L-n_{i}}(g) \subseteq l_{i}$

- reconstruct connected patterns $p_{i}=T_{L-n_{i}}^{L-n_{i}}\left(R_{g}^{\delta}\left(l_{i}^{\prime}\right)\right)$

- $\forall p_{i}$, create a node in the tree graph $t_{i} \leftrightarrow p_{i}$

- update nodes connectivity

- reiterate

$C\left\{t_{i}, t_{j}\right\}_{j<i}$

end while,

where $T_{a}^{b}$ denotes the thresholding operator between $a$ and $b, R_{g}^{\delta}(f)$ the grayscale reconstruction by geodesic dilation of $f$ inside $g$ [10, and $l_{i}$ the initial patterns extracted at resolution level $i$ (eq. 11). Note that a reconstructed pattern $p_{i}$ at resolution level $i$ will always include initial patterns $l_{j}$ of levels $j<i, p_{i} \supseteq l_{j}$, if $l_{i}$ and $l_{j}$ are either (partially) overlapping or 3D connected. In this situation, the associated nodes, $t_{i}, t_{j}$ have a parent-child relationship and a tree graph

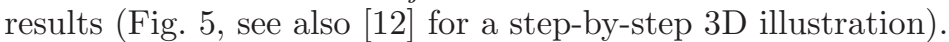

Each node $t_{i}$ of the descriptive graph carries out information related to 3D spatial location, resolution level, connected patterns, first order statistics (mean $\mu$ and standard deviation $\sigma$ ) of the native density values within $p_{i}$ pattern, $p_{i}$ volume (number of voxels), $p_{i}$ fractal dimension computed using box counting [11, $p_{i}$ relative volume with respect to its "children".

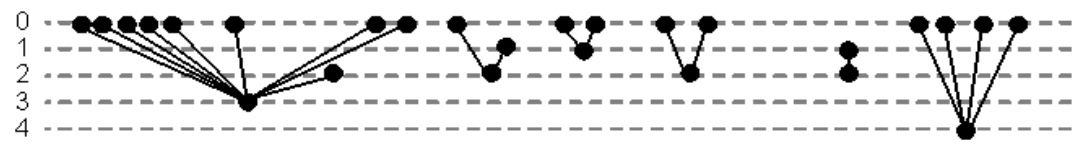

Fig. 5. Descriptive tree graph of the hierarchic relief $g$ of Fig. 3

The lung parenchyma descriptive tree graph $\left\{t_{i}\right\}_{i}$ is investigated and each node classified into normal or DPLD tissue according to a set of intuitive rules established with respect to intrinsic node properties and to those of its hierarchy [12. Three significant density classes have been defined for MDCT native data: low-density (LD) values within [-1000, -950] Hounsfield Units (HU), mediumdensity (MD) values within $(-950,-765] \mathrm{HU}$ and medium-high density (MHD) 
values within $(-765,-450]$ HU. The main structural properties of DPLDs as described in 92 are reflected in the following parameter evaluation: (1) categorize $\mu\left(t_{i}\right)$ as LD, MD or MHD; (2) categorize the $t_{i}$ children mean $\mu\left(\left\{t_{j}\right\}_{j}\right)$ as LD, MD, MHD; (3) categorize the mean of the disjoint pattern $\mu\left(t_{i} \backslash\left\{t_{j}\right\}_{j}\right)$ as LD, MD or MHD; (4) evaluate the density dispersion within the pattern: $\mu\left(t_{i}\right) / \sigma\left(t_{i}\right) \geq 3.0$ denotes a narrow dispersion, otherwise we consider a spread dispersion; (5) categorize the tissue density in the pattern (close) neighborhood; (6) estimate the pattern compactness according to the fractal dimension: $d_{f}\left(t_{i}\right)<2.0$ denotes a compact, otherwise "porous", pattern; (7) evaluate the pattern relative volume with respect to its "children" $\frac{V\left(t_{i}\right)-V\left(\left\{t_{j}\right\}_{j}\right)}{V\left(\left\{t_{j}\right\}_{j}\right)}$. After classification, each node is assigned one of the following labels: EM (emphysema), FHC (fibrosis/honeycombing), GDG (ground glass), N (normal), and the corresponding image pattern is labeled with a comprehensive color code [12].

\section{Results and Discussion}

The developed classification approach was tested on a MDCT database including 10 patients suspected of DPLD, of which 4 representative cases are illustrated Fig. 1. Their corresponding classification is illustrated Figs. 6] and 7 both locally, in axial cross-sections, and globally, by using a volume rendering approach. Here, the proximal airways are labeled distinctly by means of a region-growing procedure initiated at the top of the trachea. The pathology detection sensitivity/specificity were estimated by one experienced radiologist: EM: 94\%/80.5\%, FHC: $81.2 \% / 90 \%$, GDG: 97\%/95\%. Missclassification of EM vs. FHC occured sometimes (13\% EM classed as FHC, $22 \%$ FHC classed as EM) which also explains the low specificity value. This is due to the fact that some high-density tissues surrounding FHC patterns do not subsist in the hierarchic decomposition (Fig. 4) and, consequently, they are not considered in classification. Our future work will take into account such information together with additional features 8] for a more confident discrimination between various types of DPLDs.

To conclude, the main advances of the proposed approach with respect to the recent literature 6/78 consists in an automatic partitioning of the lung regions based on multiresolution decomposition and graph description, a fully-3D

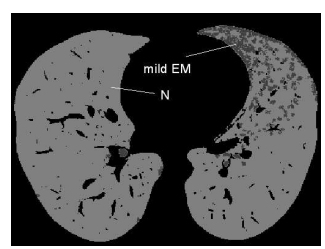

(a)

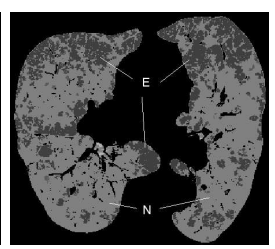

(b)

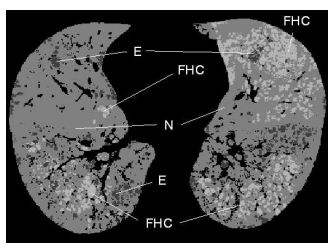

(c)

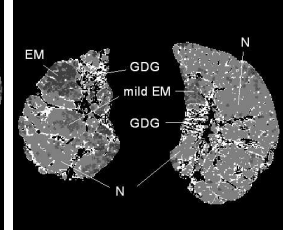

(d)

Fig. 6. Example of lung tissue classification of data from Fig. 1 (color plates in 12]) 


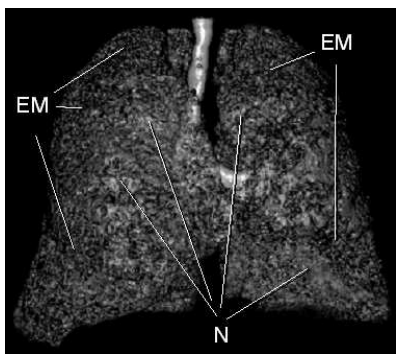

(a) Data of Fig. 6(b).

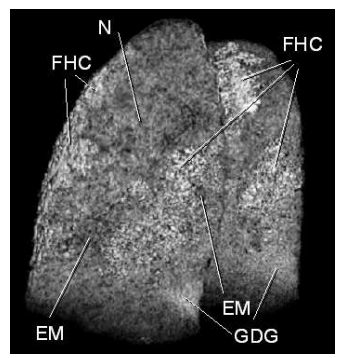

(b) Data of Fig. 6(c).

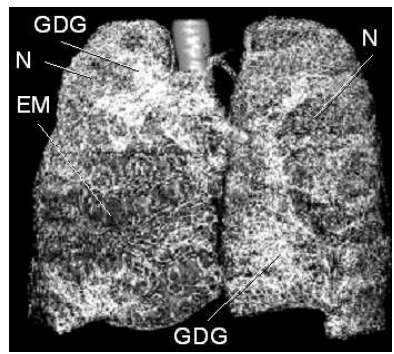

(c) Data of Fig. 6(d).

Fig. 7. Volume rendering of the lung tissue 3D classification (color plates in [12])

classification of the whole lung and the absence of training/interaction. Further improvement of the DLPD discrimination rules will be evaluated on a larger database. Note also that the rule-based pattern classification can be replaced by a Bayesian, neural network or support vector machines (SVM) approach.

\section{References}

1. Doi, K., Macmahon', H., Katsuragawa, S., Nishikawa, M.R., Jiang, Y.: Computeraided diagnosis in radiology: potential and pitfalls. Eur. J. Radiol. 31(2), 97-109 (1999)

2. Coxson, H.O., Rogers, R.M., Whittall, K.P., D'yachkova, Y., Pare, P.D., Sciurba, F.C., Hogg, J.C.: A quantification of the lung surface area in emphysema using computed tomography. Am. J. Respir. Crit. Care Med. 163(6), 1500-1501 (2001)

3. Blechschmidt, R.A., Werthschutzky, R., Lorcher, U.: Automated CT image evaluation of the lung: a morphology-based concept. IEEE Trans. Med. Imag. 20(5), 434-442 (2001)

4. Malone, J., Rossiter, J.M., Prabhu, S., Goddard, P.: Identification of disease in CT of the lung using texture-based image analysis. Proc. IEEE Conf. Signals, Systems and Computers 2, 1620-1624 (2004)

5. Xu, D.H., Kurani, A.S., Furst, J.D., Raicu, D.S.: Run-length encoding for volumetric texture. In: Proc. Visualization, Imaging and Image Processing (2004)

6. Chabat, F., Yang, G.Z., Hansell, D.M.: Obstructive lung diseases: texture classification for differentiation at CT. Radiology 228(3), 871-877 (2003)

7. Xu, Y., Sonka, M., McLennan, G., Junfeng, G., Hoffman, E.A.: MDCT-based 3D texture classification of emphysema and early smoking related lung pathologies. IEEE Trans. Med. Imag. 25(4), 463-475 (2006)

8. Xu, Y., Vanbeek, E.J.R., Yu, H., McLennan, G., Guo, J., Hoffman, E.A.: Computer-aided Classification of Interstitial Lung Disease via MDCT: 3D Adaptive Multiple Feature Method (3D AMFM). Acad. Radiol. 13(8), 969-978 (2006)

9. Fetita, C., Lucidarme, O., Preteux, F.: Automated 3D vascular segmentation in CT hepatic venography. In: Proc. SPIE Conference on Mathematical Methods in Pattern and Image Analysis, San Diego, CA, vol. 5916, pp. 114-125 (2005) 
10. Vincent, L.: Morphological gray scale reconstruction in image analysis: applications and efficient algorithms. IEEE Trans. on Imag. Proc. 2(2), 176-201 (1993)

11. Liebovitch, L.S., Toth, T.: A fast algorithm to determine fractal dimensions by box counting. Physics Letters A 141(11), 386-390 (1989)

12. Fetita, C.: Classification of lung parenchyma ROIs resulting from hierarchic decomposition. in Internal report (2007), http://www-artemis.int-evry.fr/ fetita/classif.html 